\title{
Approximate and Efficient Methods to Assess Error Fields in Spatial Gridding with Data Interpolating Variational Analysis (DIVA)
}

\author{
JEAN-MARIE BECKERS AND ALEXANDER BARTH \\ University of Liège, Liège, Belgium \\ CHARLES TROUPIN \\ IMEDEA, Esporles, Spain
}

Aida Alvera-AzCÁrATe

University of Liège, Liège, Belgium

(Manuscript received 11 June 2013, in final form 28 August 2013)

\begin{abstract}
This paper presents new approximate methods to provide error fields for the spatial analysis tool Data Interpolating Variational Analysis (DIVA). The first method shows how to replace the costly analysis of a large number of covariance functions with a single analysis for quick error computations. Then another method is presented where the error is only calculated in a small number of locations, and from there the spatial error field itself is interpolated by the analysis tool. The efficiency of the methods is illustrated on simple schematic test cases and a real application in the Mediterranean Sea. These examples show that with these methods, one has the possibility for quick masking of regions void of sufficient data and the production of "exact" error fields at reasonable cost. The error-calculation methods can also be generalized for use with other analysis methods such as three-dimensional variational data assimilation (3DVAR) and are therefore potentially interesting for other implementations.
\end{abstract}

\section{Introduction}

Spatial analysis of observations, also called gridding, is a common task in oceanography and meteorology, and a series of methods and implementations exists and is widely used. Here $N_{d}$ data points of values $d_{i}$, $i=1, \ldots, N_{d}$ at location $\left(x_{i}, y_{i}\right)$ are generally distributed unevenly in space. Furthermore, the values of $d_{i}$ are affected by observational errors, including representativity errors. From this dataset an analysis on a regular grid is often desired. It has been quickly recognized that it would be natural to define the best analysis as the one that has the lowest expected error. This definition has led to kriging and optimal interpolation (OI) methods (e.g., Gandin 1965; Delhomme 1978; Bretherton et al. 1976) and to the Kalman-Bucy filter and data

Corresponding author address: Jean-Marie Beckers, GHERAGO, University of Liège, Sart-Tilman B5, 4000 Liège, Belgium. E-mail: jm.beckers@ulg.ac.be assimilation with adjoint models in the context of forecast models (e.g., Lorenc 1986).

These methods assume that statistics on observational errors and the spatial covariance of the field to be analyzed are available to infer the "best" analysis field. As these methods aim at minimizing the analysis error, it is not a surprise that they also provide the theoretical a posteriori error field for the analysis. The practical implementation of these methods can lead to very different performances, also when it is necessary to calculate the error fields (e.g., Bouttier and Courtier 2002).

The present paper will focus on a computationally efficient way to provide error fields for a gridding tool called Data Interpolating Variational Analysis (DIVA), whose full description can be found elsewhere (Brasseur 1994; Brasseur et al. 1996; Troupin et al. 2012) and is not repeated here. Looking at DIVA gridding is not restrictive, as we can later exploit relationships with other formulations to allow for generalizations. In DIVA, the gridded field $\varphi$ over the two-dimensional domain $D$ is searched as the field that minimizes $J$, defined as 


$$
J(\varphi)=\sum_{i=1}^{N_{d}} \mu_{i}\left[d_{i}-\varphi\left(x_{i}, y_{i}\right)\right]^{2}+\|\varphi\|^{2},
$$

where weights $\mu_{i}$ control how the analysis has to be close to the data $d_{i}$ and where the norm $\|\varphi\|$ measuring spatial irregularity is defined as

$$
\|\varphi\|=\int_{D}\left(\alpha_{2} \nabla \nabla \varphi: \nabla \nabla \varphi+\alpha_{1} \nabla \varphi \cdot \nabla \varphi+\alpha_{0} \varphi^{2}\right) d D .
$$

This term enforces the solution to be more or less regular via the use of the gradient operator $\nabla=(\partial / \partial x, \partial / \partial y)$. Coefficients $\alpha_{2}, \alpha_{1}$, and $\alpha_{0}$ control to what extent curvature, gradients, and amplitudes of the fields are penalized. ${ }^{1}$ The term penalizing means that the amplitude of the solution ensures that in regions far away from data, the analyzed anomalies tend to zero, which avoids the extrapolation problems one would otherwise encounter (e.g., Seaman and Hutchinson 1985). The parameters of the formulation, which can be translated into a correlation length scale and a signal-to-noise ratio (e.g., Brasseur et al. 1996), can be calibrated by crossvalidation techniques such as described in Wahba and Wendelberger (1980). However, the highest order (two) of the derivatives in the regularization term remain fixed, as the other parameters allow for sufficient freedom.

This formulation is discretized on a finite-element mesh covering the domain with triangles. Each of the triangles is in fact subdivided into three subtriangles on each of which the solution is expanded as a cubic polynomial. This rich function allows a sufficient degree of continuity so that the functional is well defined. The unknowns are then the coefficients of the polynomials, or in the finite-element vocabulary, the connectors. The functional is a quadratic function of these connectors, and the minimization leads to a linear system to be solved for these connectors. In the present implementation, this solution is done by a direct skyline solver exploiting the banded structure of the matrix to invert. For larger problems the recent DIVA version also allows for an iterative solution of this sparse linear system with a preconditioning.

Because of the finite-element grid covering only the real domain of interest $D$, disconnections, barriers, islands, etc. are naturally taken into account (e.g., Troupin et al. 2010). The solution can be shown to be equivalent to an optimal interpolation (e.g., McIntosh 1990; Barth et al. 2013) and to the solution of another minimization

\footnotetext{
${ }^{1}$ The equation a $\cdot \mathbf{a}$ stands for the standard scalar product $\sum_{i} a_{i} a_{i}$ of vectors and $\mathbf{A}: \mathbf{A}$ is for its generalization, $\sum_{i, j} A_{i j} A_{i j}$.
}

problem, where the function to be minimized is defined as

$$
2 J(\mathbf{x})=\left(\mathbf{x}-\mathbf{x}_{b}\right)^{\mathrm{T}} \mathbf{B}^{-1}\left(\mathbf{x}-\mathbf{x}_{b}\right)+(\mathbf{H} \mathbf{x}-\mathbf{d})^{\mathrm{T}} \mathbf{R}^{-1}(\mathbf{H} \mathbf{x}-\mathbf{d}),
$$

where $\mathbf{x}$ is a column array storing the analyzed field on each grid point where the analysis is needed; $\mathbf{d}$ is an array containing the observations; and $\mathbf{H}$ is a linear observation operator that extracts the gridded solution at the data locations, so that $\mathbf{d}-\mathbf{H} \mathbf{x}$ measures the misfit between the observations and the field $\mathbf{x}$. The covariance matrix $\mathbf{B}$ is of the background field $\mathbf{x}_{b}$, and $\mathbf{R}$ is a covariance matrix holding the observational error covariances. The equivalence with DIVA is ensured if $\mathbf{R}$ is diagonal and $\mathbf{B}$ is using the so-called kernel of the norm (2) as a covariance function. The kernel is in fact nothing but the correlation function one would use to create $\mathbf{B}$, yielding the same result in OI as with the variational approach (e.g., Wahba and Wendelberger 1980). Furthermore, for an equivalent result the weights $\mu_{j}$ are scaled by the inverse of the signal-to-noise ratio defined by matrices $\mathbf{B}$ and $\mathbf{R}$ (Barth et al. 2013). The minimization formulation (3) is a special case of the so-called threedimensional variational data assimilation (3DVAR) method (e.g., Fischer et al. 2005) with a linear observation operator. For simplicity we keep the name 3DVAR even if we use the equivalence with DIVA in a 2D framework. The solution that minimizes the 3DVAR functional (3) is itself equivalent to the OI analysis step (e.g., Kalnay 2003), defined as

$$
\mathbf{x}-\mathbf{x}_{b}=\mathbf{K}\left(\mathbf{d}-\mathbf{H} \mathbf{x}_{b}\right)
$$

with the Kalman gain matrix $\mathbf{K}$ defined as

$$
\mathbf{K}=\mathbf{B} \mathbf{H}^{\mathrm{T}}\left(\mathbf{H B H ^ { \mathrm { T } }}+\mathbf{R}\right)^{-1} \text {. }
$$

The choice of the background field $\mathbf{x}_{b}$ depends on the application: for operational forecasts, it is the modeled forecast; for oceanic cruise mapping, it can be a climatological field; for the computation of climatologies, it can be a constant reference value, etc. For the simplicity of the presentation, assume from here on that we work with anomalies with respect to this background field ( $\mathbf{x}-\mathbf{x}_{b}$ is replaced by $\mathbf{x}$ and $\mathbf{d}-\mathbf{H} \mathbf{x}_{b}$ is replaced by $\mathbf{d}$ ).

The analysis-error covariance $\mathbf{P}$ then reads with different equivalent formulations (e.g., Rabier and Courtier 1992; Courtier et al. 1994; Bouttier and Courtier 2002) as shown:

$$
\mathbf{P}=\left(\mathbf{B}^{-1}+\mathbf{H}^{\mathrm{T}} \mathbf{R}^{-1} \mathbf{H}\right)^{-1}=\mathbf{B}-\mathbf{K H B} .
$$


As easily seen, this matrix is also the inverse of the Hessian $^{2}$ matrix of $J$ in (3). The diagonal terms of the error covariance matrix provide the error variance of the analysis on the grid defined by $\mathbf{x}$. This error variance in each point is the quantity we will focus on later.

From (6), between the data locations, the error covariance $\mathbf{P}_{d}$ of the analysis is expressed in terms of the background covariance matrix between data locations $\mathbf{B}_{d}=\mathbf{H B H}^{\mathbf{T}}$ :

$$
\begin{aligned}
\mathbf{P}_{d} & =\mathbf{H} \mathbf{P} \mathbf{H}^{\mathrm{T}}=\mathbf{H B H}^{\mathrm{T}}-\mathbf{H} \mathbf{K H B H} \mathbf{H}^{\mathrm{T}} \\
& =\mathbf{B}_{d}-\mathbf{B}_{d}\left(\mathbf{B}_{d}+\mathbf{B}\right)^{-1} \mathbf{B}_{d}=\mathbf{B}_{d}\left(\mathbf{B}_{d}+\mathbf{R}\right)^{-1} \mathbf{R} .
\end{aligned}
$$

In DIVA or 3DVAR, matrices $\mathbf{K}$ and $\mathbf{B}$ are actually never formed, but the application of $\mathbf{K}$ to a vector can be seen as the application of the analysis tool to a dataset stored in this vector. Similarly HK applied to a vector consists of applying the tool to the data and then retrieving the analysis at the data locations.

For the linear observation operators used here, DIVA, 3DVAR, and OI provide the same results (under the hypotheses mentioned above), but the computational aspects are quite different, in particular when it comes to the error calculations.

For 3DVAR implementations, the calculation of the a posteriori error covariance requires the computation of the inverse Hessian matrix, whereas the analysis itself only uses gradient calculations (e.g., Rabier and Courtier 1992). To some extent, the need to calculate the full Hessian matrix can be circumvented by the use of Lanczos vectors of the conjugate gradient approach (e.g., Moore et al.2011, in the context of 4DVAR). However, in this case the need of more Lanczos vectors required to provide an accurate estimate of the Hessian matrix defeats the purpose of the conjugate gradient approach to use as few iterations as possible. More recently, with approaches specifying the background covariance matrices by an ensemble (e.g., Hamill and Snyder 2000), error calculations can use the equivalence with OI to exploit the reduced rank of the covariance matrix.

For OI, in each point where the analysis is needed, an analysis of the covariance is requested for the a posteriori error calculation. This can lead to very high computational costs unless reduced rank approaches are possible (e.g., Kaplan et al. 2000; Beckers et al. 2006) or localization is used (e.g., Reynolds and Smith 1994). In the latter case, the error field can be calculated at the same time as the local analysis at almost no additional

\footnotetext{
${ }^{2}$ Derivatives are with respect to coordinate $\mathbf{x}$.
}

cost. It also has the advantage of allowing a highly parallel approach.

For DIVA, several problems exist: 1) neither covariance functions nor background matrices are explicitly formulated, so that error calculations have been only made possible by exploiting the equivalence with $\mathrm{OI}$ and the discovery of a quick method to numerically calculate on the fly covariance functions (Troupin et al. 2012); 2) the computational burden is still high, as an analysis in each of the $N$ points where the error is requested must be performed; 3) localization could only be exploited at the inversion step of the finite-element formulation by exploiting the banded structure of the matrix to calculate the value of a connector. This has not been implemented, as it would lead to suboptimal solutions and in any case would not allow the error calculation in parallel with the analysis (such as in OI implementations), as the error field is not formulated in terms of connectors.

So, several methods are faced with high computational costs to retrieve error fields. Because covariances are generally estimated from data (e.g., Emery and Thomson 2001) and are not perfectly specified, we expect that error fields derived from the theoretical models are not "true" error fields in any case. Therefore, it can be considered overkill in computations trying to calculate errors with the full theoretical formulation in all locations and some relaxation can be accepted.

The present paper will present two "error calculations" in section 2 that to various degrees mimic the "exact" error field but with reduced cost. The method will be illustrated in section 3 with the 2D version of DIVA, but generalizations to the other cases mentioned in the introduction will be discussed in section 4 .

\section{Approximations for error analysis at reduced costs}

The direct formulations for error covariances are rarely applied because matrices are too large and/or covariance matrices are not explicitly formulated. Alternative ways to get information on the analysis error are desirable.

If we are only interested in the trace of $\mathbf{P}$, providing a global error estimate, randomized estimates (e.g., Girard 1998) apply the analysis tool to random vectors and provide trace estimates (e.g., Troupin et al. 2013, manuscript submitted to Geosci. Model Dev. Discuss.). These methods converge quite easily and are used in cross-validation techniques (e.g., Xiang and Wahba 1996). The estimation of each individual term on the diagonal, however, is more challenging and convergence is much slower. It is possible to use particular structures in the random vectors (e.g., Bekas et al. 2007), but 
convergence turns out to be still rather slow for our case, needing a number of analyses of random vectors to be performed not significantly lower than $N$, the number of diagonal terms to be evaluated. Here we will exploit the idea of applying the analysis tool not to randomly chosen "data vectors" but to well-designed ones. This has been implemented to probe the diagonal (Tang and Saad 2012), but here we will try to capture only some of the diagonal terms and then guessing the other terms by spatial coherence. Instead of trying to calculate the error covariance, we can focus on the error reduction term $\boldsymbol{\Delta}$ defined from (6) by $\mathbf{P}=\mathbf{B}-\boldsymbol{\Delta}$ :

$$
\boldsymbol{\Delta}=\underbrace{\mathbf{B} \mathbf{H}^{\mathrm{T}}\left(\mathbf{H B H}^{\mathrm{T}}+\mathbf{R}\right)^{-1}}_{\mathbf{K}} \mathbf{H B} .
$$

This formulation shows that if we have a tool to analyze a data array (the underbraced terms are the formal equivalent of the tool), it is sufficient to analyze covariances (columns of $\mathbf{H B}$ ) to get the error field, but for each point in which the error is requested another covariance must be analyzed. For DIVA, the main challenge in the past was the fact that covariances are never explicitly formulated, yet needed for the error computation. In the previous DIVA versions (e.g., Rixen et al. 2000), this problem was circumvented by using as an approximate covariance function an analytical solution for the minimum of (1) applied in an infinite isotropic domain (a method called hybrid in the following and used in Brankart and Brasseur 1996; Troupin et al. 2012). Recently, Troupin et al. (2012) showed how to use the DIVA tool itself to numerically calculate the covariances in an optimized way (a method called real covariance in the following). Now we will aim at downgrading this method to make it more economical.

\section{a. Clever poor man's error}

If we replace covariances to be analyzed with a vector with all elements being a constant background variance, then we generally overestimate the error reduction but we have a computational huge gain, because the same analysis is valid for ALL of the $N$ points in which we want to calculate the error. Instead of $N$ backward substitutions or iterative solutions, we only need one additional analysis to add an "error field" to the analysis. This was already implemented (Troupin et al. 2010) and was called poor man's error. In reality we can do better for a similar cost by looking at the situation of an isolated data point and focusing on the error reduction (8).

With a single data of anomaly value $d$ and isotropic covariances, the analysis $x^{a}$ at a distance $r$ from the data location reads according to (4):

$$
x^{a}(r)=\frac{\sigma^{2}}{\sigma^{2}+\epsilon^{2}} c(r / L) d,
$$

where $c(r / L)$ is the correlation function of the background field (the kernel of the DIVA functional); $\epsilon^{2}$ is the observational noise; and $\sigma^{2}$ is the variance of the background field, defining the so-called signal-to-noise ratio $\sigma^{2} / \epsilon^{2}$. The error reduction term (8), scaled by the background variance, reads

$$
\frac{\Delta(r)}{\sigma^{2}}=\frac{\sigma^{2}}{\sigma^{2}+\epsilon^{2}} c^{2}(r / L)
$$

We see that when applying the idea of the poor man's error (putting $d=1$ into the analysis) analysis, (9) yields some resemblance to the actual nondimensional error reduction (10), but it overestimates the error reduction since it uses $c$ instead of $c^{2}$.

To go further, we can notice that for the often used Gaussian correlation $c=\exp \left(-r^{2} / L^{2}\right)$, we have $c^{2}(r / L)=$ $c(\sqrt{2} r / L)$. In other words, in this case we can obtain the exact error reduction by applying the poor man's error approach with a length scale divided by $\sqrt{2}$. For more general correlation functions, obviously it is rare to find a new length scale $L^{\prime}$ such that $c\left(r / L^{\prime}\right)=c^{2}(r / L)$, but one can try to optimize the value so that the functions are close to each other in a root-mean-square sense in a $2 \mathrm{D}$ domain:

$$
\min _{L^{\prime}} \int_{0}^{\infty}\left[c\left(r / L^{\prime}\right)-c^{2}(r / L)\right]^{2} r d r
$$

This minimization can be done easily when the covariance function is known. For DIVA, the covariance function in an infinite 2D domain can be expressed in terms of the modified Bessel function $K_{1}$ as (Brasseur 1994)

$$
c(r / L)=\frac{r}{L} K_{1}\left(\frac{r}{L}\right)
$$

and the optimal value of $L^{\prime}$ is

$$
L^{\prime}=\frac{L}{1.70677}
$$

The quality of the approximation can be seen in Fig. 1.

If we have several data points separated by distances much larger than the correlation length scale, then the presence of other data points does not influence the analysis and error field around a data point and hence the poor man's error calculation replacing all data values by one and changing the correlation length scale will provide, with a single analysis, the error reduction term on the full grid. 


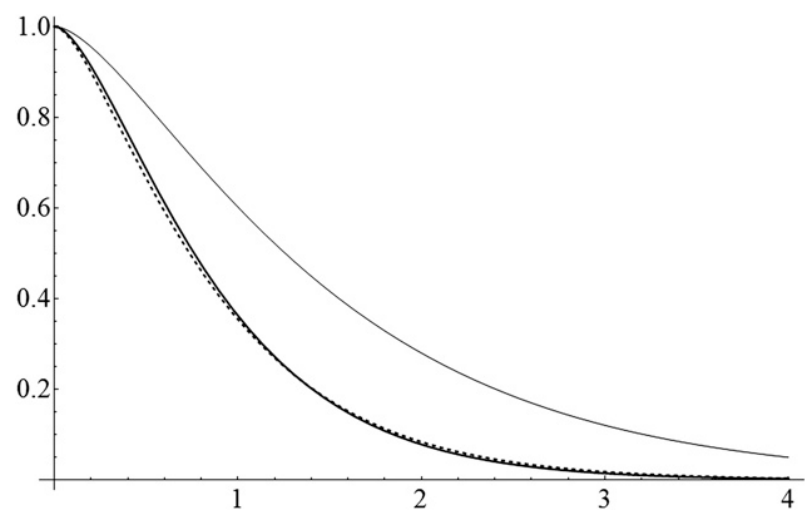

FIG. 1. DIVA correlation function (the kernel of the DIVA functional) in an infinite domain as a function of $r / L$ (thin line). The squared correlation function that leads to the exact error reduction for one data point (thick line) shows how strongly the poor man's error using the thin line overestimates the error reduction. Adapting the correlation length scale $c\left(r / L^{\prime}\right)$ (dashed line) in the poor man's error (called clever poor man's error) shows how one can mimic the exact squared correlation by comparing the thick and dashed lines.

For regions with higher data coverage, the method provides a too optimistic view of the error, but the method can be easily used to mask gridded regions far away from the data (see error on mask on a $101 \times 101$ grid in Fig. 2).

The recipe for the method, which we call clever poor man's error, is thus straightforward: adapt the correlation length scale and then apply the analysis tool to a data vector with unit values to retrieve the complete error reduction field in a single analysis step.

\section{b. Almost exact error fields}

For a diagonal observational error covariance matrix, the error at data location $i$ is easily reformulated from (7) as

$$
\mathbf{e}_{\mathbf{i}}^{\mathrm{T}} \mathbf{P}_{d} \mathbf{e}_{\mathbf{i}}=R_{i i} \mathbf{e}_{\mathbf{i}}^{\mathrm{T}} \mathbf{B}_{d}\left(\mathbf{B}_{d}+\mathbf{R}\right)^{-1} \mathbf{e}_{\mathbf{i}}
$$

where $\mathbf{e}_{\mathbf{i}}$ is a vector with zeros everywhere except 1 on element $i$. This makes an interesting parameter appear:

$$
A_{i i}=\mathbf{e}_{\mathbf{i}}^{\mathrm{T}} \mathbf{B}_{d}\left(\mathbf{B}_{d}+\mathbf{R}\right)^{-1} \mathbf{e}_{\mathbf{i}}=\mathbf{e}_{\mathbf{i}}^{\mathrm{T}} \underbrace{\mathbf{H} \mathbf{H}^{\mathrm{T}}\left(\mathbf{H B H}^{\mathrm{T}}+\mathbf{R}\right)^{-1}}_{\mathbf{K}} \mathbf{e}_{\mathbf{i}} .
$$

If we know the value of $A_{i i}$, then the error is readily available at the data location as $R_{i i} A_{i i}$. As before, the calculation of $A_{i i}$ is suggested by the formulation read from right to left: It is sufficient to apply the analysis tool (K) to a data vector with zeros everywhere and 1 at location $i$ (vector $\mathbf{e}_{\mathrm{i}}$ ) and then taking the value of the analysis at location $i$ (operator $\mathbf{e}_{\mathbf{i}}^{\mathrm{T}} \mathbf{H}$ ).

There is, in fact, another reason to calculate $A_{i i}$ : it provides a way for a data quality check. Indeed (e.g., Bretherton et al. 1976; Troupin et al. 2013, manuscript submitted to Geosci. Model Dev. Discuss.), the expected misfit between observation and analysis $\varepsilon[\mathbf{d}-\mathbf{H K d}]$ has the following variance:

$$
\varepsilon\left[(\mathbf{d}-\mathbf{H K d})(\mathbf{d}-\mathbf{H K d})^{\mathrm{T}}\right]=\mathbf{R}(\mathbf{I}-\mathbf{H K}) .
$$

This variance can be exploited to check whether the actual analysis-data difference is significantly different from the expected difference, which then allows one to flag data as suspect. At data location $i$ with a diagonal observational error, the data-analysis misfit according to (16) should have the following variance:

$$
s_{i}^{2}=R_{i i}\left(1-A_{i i}\right),
$$

so that comparing the actual analysis-data misfit to the expected one, suspect data can be identified.

For this use and also because $A_{i i}$ is needed in crossvalidation techniques (e.g., Wahba and Wendelberger 1980; Brankart and Brasseur. 1996), the calculation of $A_{i i}$ (via an analysis of a data vector with zeros everywhere except at data point $i$ ) has been optimized for DIVA and is accessible at reasonable cost (Troupin et al. 2013, manuscript submitted to Geosci. Model Dev. Discuss.). This means we can calculate the error estimates at data locations, which leaves only one problem, how do you calculate the error in other locations?

An easy way to achieve this is to add a pseudodata point with a virtual huge observational error for any location where the error has to be calculated. For DIVA, this high observational error translates into a very small data weight $\mu_{i}$ (1) that numerically does not cause any problem in the data analysis step. It is then easy to calculate the error at any location. However, this would still be costly if done everywhere, as $A_{i i}$ needs to be calculated in this pseudodata location without the benefit in terms of outlier detection or cross validation (as we know that the data are not real). We should therefore limit the number of additional pseudodata points and still be able to calculate the error everywhere. In fact, we can consider this again as a gridding problem: knowing the error "exactly" at a series of points, what is the value of the error field in other locations? We can thus use the gridding tool itself, where the "observations" are the calculated errors and where the "observational" error is zero and hence the signal-to-noise ratio is infinity (or just very large in the numerical code). There remains to specify the correlation length scale for gridding the error 

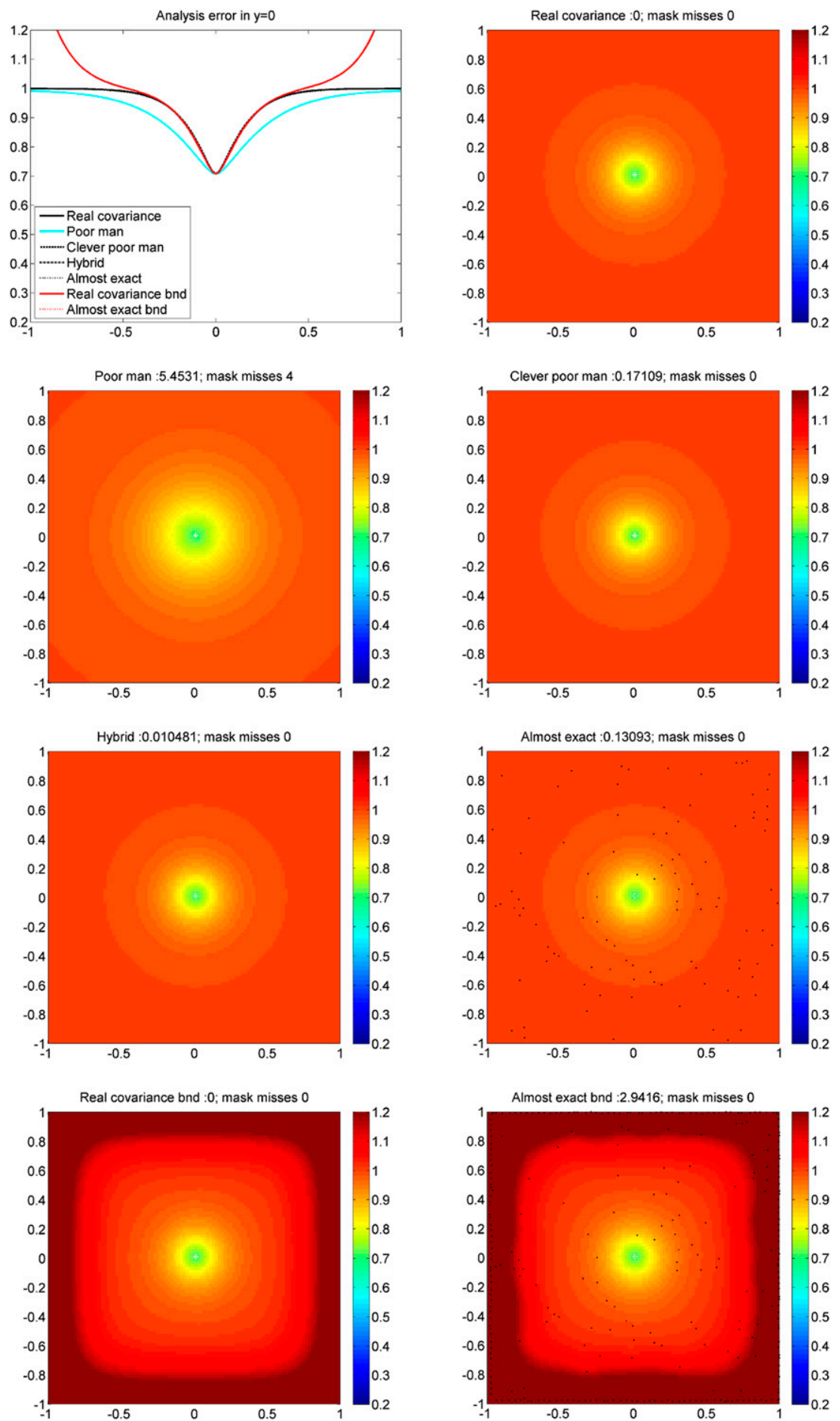

FIG. 2. Test case with a single point in the center of the domain. The error standard deviation is shown for the different methods. (top-left) A section along $y=0$. The title for each $2 \mathrm{D}$ plot identifies the method and includes two indicators of the quality of the error field. The first number is the relative error on the error field as a percentage, where the true field is the field real covariance when the error is scaled by the local background variance. For the case where boundary effects are taken into account, the reference solutions is real covariance bnd. The second indicator gives the number of grid points where a mask derived from the error field is not the same as the exact one. White crosses indicate real data locations, and black dots indicate pseudodata locations. 
field, but as shown in the analysis of the clever poor man's error, a good choice is the adapted length scale $L^{\prime}$ (13). Furthermore, it is easy to define the background field if we grid the error reduction: since the "data" locations are the places where we have the error exactly, in other locations we do not have data and the background error reduction is simply zero. Finally, because of the influence of data over a correlation length distance, it seems reasonable to add randomly $\alpha^{2} D / L^{2}$ pseudodata over the surface $D$, where $\alpha \sim 1$ defines the precision with which we want the error field.

For completeness, a discussion on the background covariance is needed. Up to now we have scaled the error reduction by $\sigma^{2}$, the overall background variance. However, with DIVA, the background covariance varies spatially and increases near boundaries because of the variational formulation (Troupin et al. 2012). So, the local background variance in location $(x, y)$ has a value of $\sigma^{2} \hat{B}(x, y)$, where $\hat{B}(x, y)$ is now a nondimensional local background covariance. Sometimes it is interesting to present the relative errors, in which case a scaling by this local background covariance is necessary. The calculation of local background variances can be done at some cost with the covariance module (Troupin et al. 2012) of DIVA, but only applied in the data points in this case. So, one has the choice to scale or not with this nondimensional background field, and the unscaled error field is referred to as with boundary effect (bnd). With the boundary effect, because of the less uniform behavior of the error field near the boundaries (see examples later), we generate a series of pseudodata in each finite-element mesh forming the boundary (we can add more points compared to the scaled error field because we do not need to calculate the local background variance in the unscaled version).

A final comment concerns the number of data and the cost to calculate $A_{i i}$ for each data point: generally the number of data points is much lower than the number of grid points, so that the computational burden to calculate these coefficients remains reasonable compared to the burden of a full error calculation. Should there be a very large number of observations, there is no problem to restrict the error calculation to a subset of the data points, as together with the pseudodata points a nice coverage of the grid is easily achieved.

\section{Test cases}

To diagnose the quality of the error estimates, we will provide three indicators: a graphical representation and two numbers. The first metric is simply the relative error on the error field (the root-mean-square of the difference in error variances between the true error field and the approximate one compared to the true error variance). The second one tries to check how well the error field can be used to mask regions with insufficient data coverage. Typically, when the error variance of the analysis is larger than $50 \%$ of the background variance, it means the data did not provide a significant amount of information and the analysis could be masked. Then we can compare the masks derived from the exact error and the approximate one and see how many grid points do not have the same mask.

\section{a. A single data point}

This case simply serves to check that the analysis we showed is valid and to see how the different methods compare in the situation with a single data point in the center of the domain with a unit signal-to-noise ratio and a unit background variance. In this case, the error variance at the origin is 0.5 and the standard deviation shown in Fig. 2 is 0.707 . The number of grid points for the gridded field is $101 \times 101$ to which we can compare the number of mask misses.

For all errors without taking into account the boundary effects, the visual inspection shows that the hybrid, the clever poor man's error, and the almost exact error approach are indistinguishable from the exact solution. Only the poor man's error is significantly different, as expected. Quantitatively, the relative errors on the error fields are less than a percent and no mask errors occur, except again for the poor man's error. The hybrid error estimate is very close to the exact one using real covariances. The slight difference is because the analytical covariance function (12) is the one of an infinite domain, whereas the computation domain used here is finite. When boundary effects are taken into account, we observe the highest errors near the boundary [see Troupin et al. (2012) for details and explanations]. But again, the approximate fields are of excellent quality, though with higher rms error because of the stronger spatial variability of the error field. To capture this variability better, we can increase the number of pseudodata by increasing $\alpha$. Indeed, with a value of $\alpha=3$ (Fig. 3) the quality increases, whereas decreasing the value of $\alpha$ provides still acceptable results and the error mask is still excellent in this case.

The computational time, not yet shown here as a single data point, is rarely encountered in practice and the CPU time of the present case is similar to the one in section $3 \mathrm{c}$ (see Table 1).

\section{b. Aligned data points}

A slightly more complicated situation is one where 10 points are aligned in $y=0$ for $x \geq 0$ as shown in Fig. 4. 

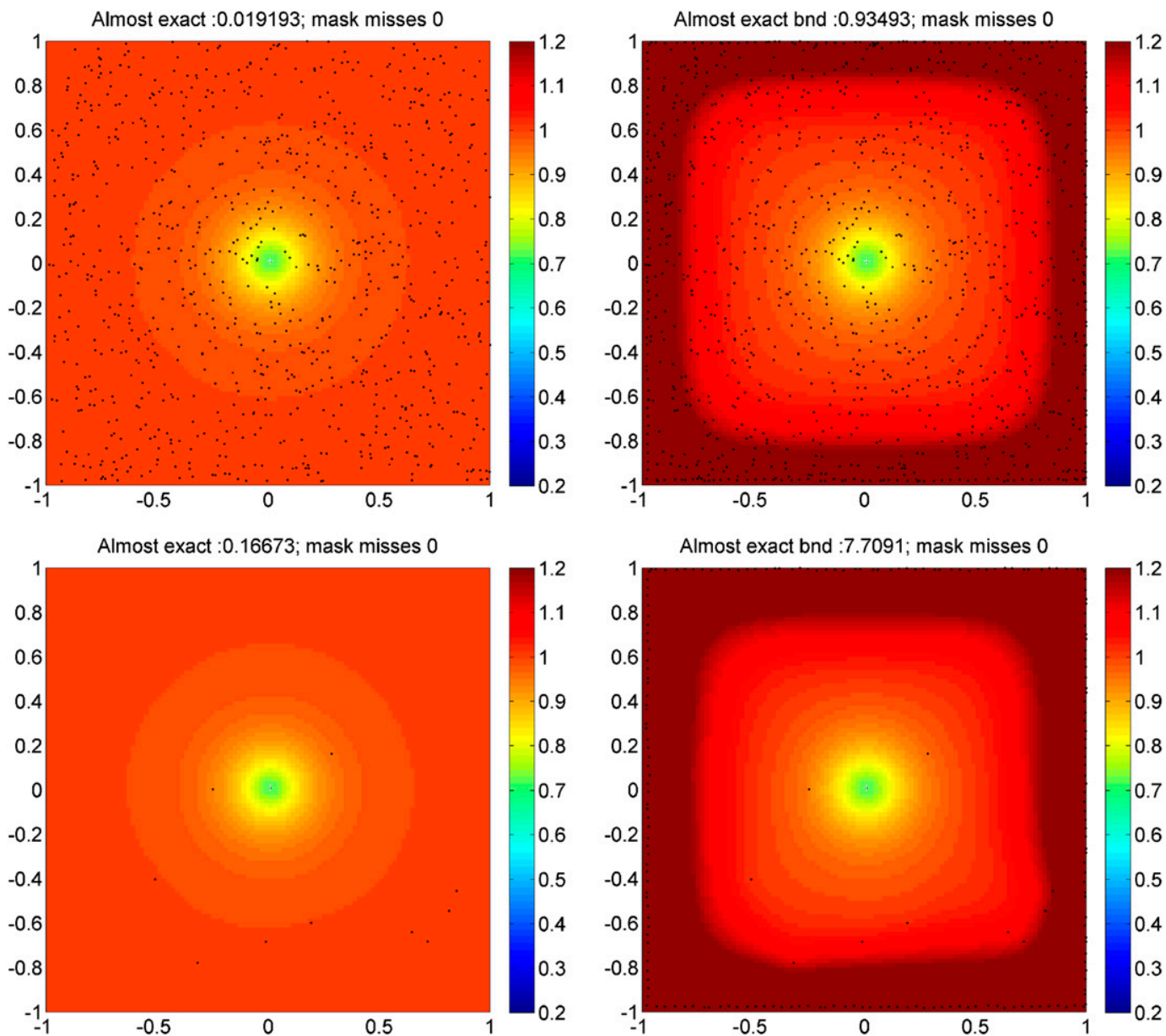

FIG. 3. Error fields for a single point in the center with (top) fine sampling $\alpha=3$ of pseudodata and (bottom) coarse sampling $\alpha=0.3$. White crosses indicate real data locations, and black dots pseudodata locations.

The poor man's error is now clearly too optimistic, also at the data locations, because it overestimates the error reduction at each data point due to the other data points. The clever poor man's estimate clearly reduces the problem, but the hybrid and the almost exact error outperform it. We also see that the hybrid method degrades near data points close to the boundary, as to be expected.

\section{c. Points in part of the domain only}

The same conclusions as in the previous case hold if we now place 150 data points in the top-right part of the domain (Fig. 5). The clever poor man's error improves the results from the poor man's error, but the hybrid and the almost exact error perform better, with the best approximate method again the almost exact error version. For boundary effects, capturing the error field near the boundary is more problematic but the error field and mask are still of quality.
Up to now we only compared the quality of the fields, but we can also compare the computational load. As seen in Table 1, the most expensive methods are those calculating the exact field (with scaled or unscaled background variances). The hybrid method consumes less time because it does not need the calculation of a covariance function by another DIVA calculation but

TABLE 1. CPU time for the test case with 150 data points distributed randomly in part of the domain (schematic case) and a realistic case of the Mediterranean Sea.

\begin{tabular}{lcc}
\hline \multicolumn{1}{c}{ Method } & Schematic case (s) & Realistic case (s) \\
\hline Real covariance & 572.4 & 1944.9 \\
Poor man & 3.8 & 6.1 \\
Clever poor man & 8.3 & 13.4 \\
Hybrid & 345.3 & 1256.6 \\
Almost exact & 37.2 & 81.4 \\
Real covariance bnd & 568.3 & 1951.9 \\
Almost exact bnd & 33.7 & 175.1 \\
\hline
\end{tabular}



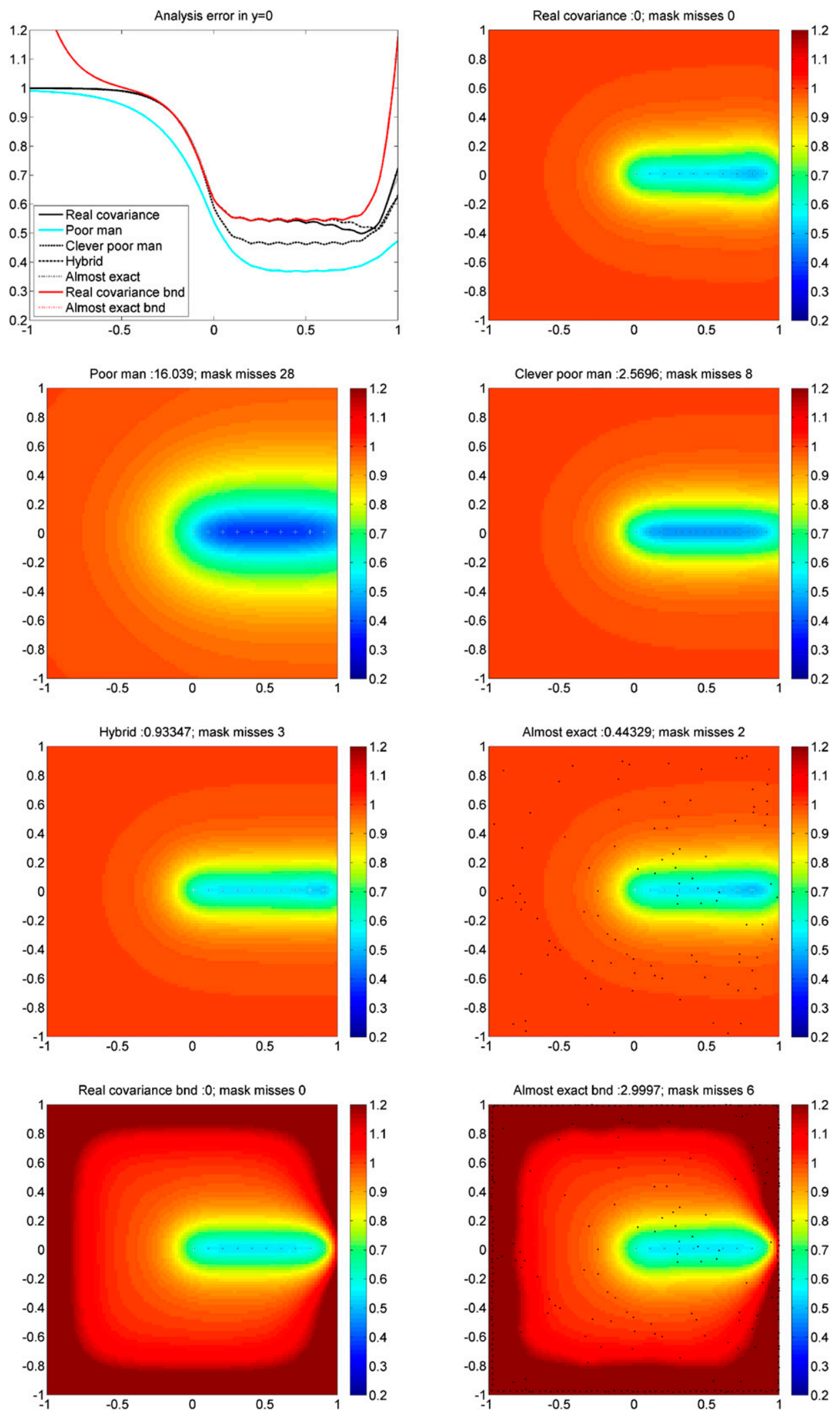

FIG. 4. Error fields for 10 data points in $y=0, x \geq 0$. White crosses indicate real data locations, and black dots pseudodata locations. 

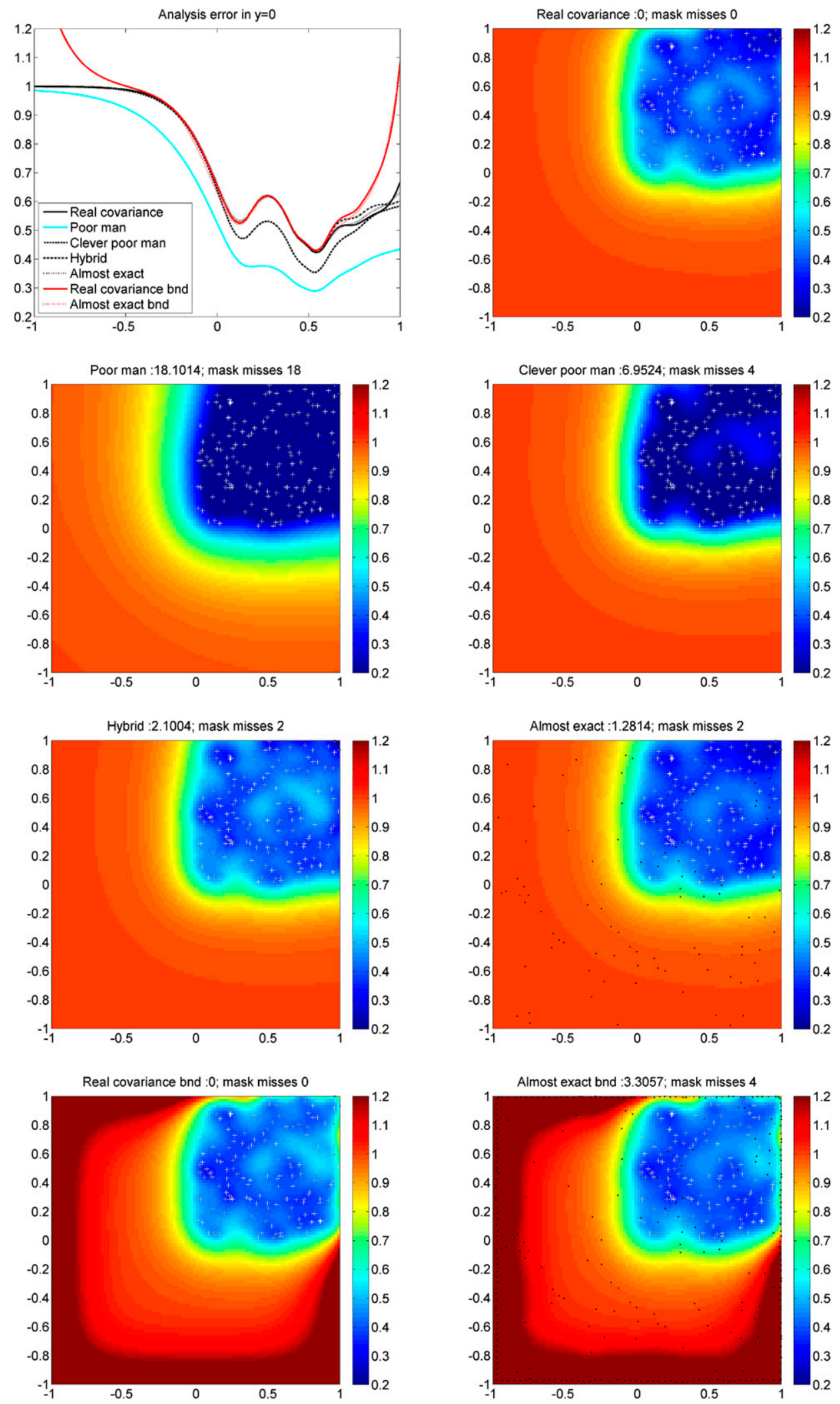

FIG. 5. Error fields for 150 random points in one quadrant. White crosses indicate real data locations, and black dots pseudodata locations. 


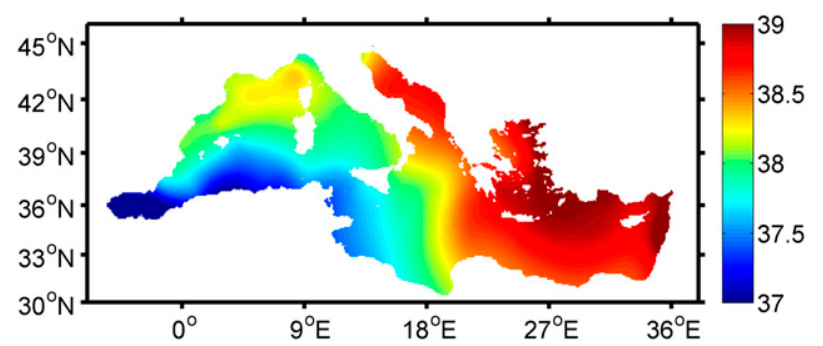

FIG. 6. Analysis of salinity measurements in the Mediterranean Sea at a depth of $30 \mathrm{~m}$ in July for the 1980-90 period.

can use an analytical function instead. However, compared to the cost of the almost exact error version, the hybrid method is one order of magnitude more expensive, yet the almost exact error calculation provides error estimates of similar or better quality. Finally, the poor man's error calculations are clearly the fastest and therefore interesting for exploratory work.

\section{d. Realistic test case}

We finally test the methods with the same dataset as the one used in Troupin et al. (2012) so that we can use the same statistical parameters and do not need to recalibrate the analysis. We use salinity measurements in the Mediterranean Sea at a depth of $30 \mathrm{~m}$ in July, for the 1980-90 period and reconstruct the solution on a highresolution output grid with $500 \times 250$ grid points.

The analysis itself (Fig. 6) shows the well-known features such as the inflow of Atlantic waters in Gibraltar; the anticyclonic gyres in the Alboran Sea; the spreading of the Atlantic waters off the North African coast; the high salinities of the eastern Levantine Basin, a signature of Black Sea waters in the Aegean Sea; and the high salinity in the northern part of the western Mediterranean. Also the influence of the Po River in the northern Adriatic is visible. This analysis itself is calculated within a few seconds, and we focus now on the computationally more expensive error fields.

The error fields are scaled by the global background variance, and white crosses indicate real data locations and black dots indicate pseudodata locations. The real error field (top panel of Fig. 7) shows the effect of low data coverage in the southern parts and the lower errors near data locations. As before, the poor man's error is quite optimistic but quantitatively not reliable. The mask derived from the poor man's error with only 43 incorrectly masked points has some skills, but the clever poor man's error provides more acceptable quantitative results and masks. In particular, the regions void of data in the southern part and around Sardaigna are now captured. The hybrid method and the almost exact approach (Fig. 8) have similar metrics, but if we look at the details, the
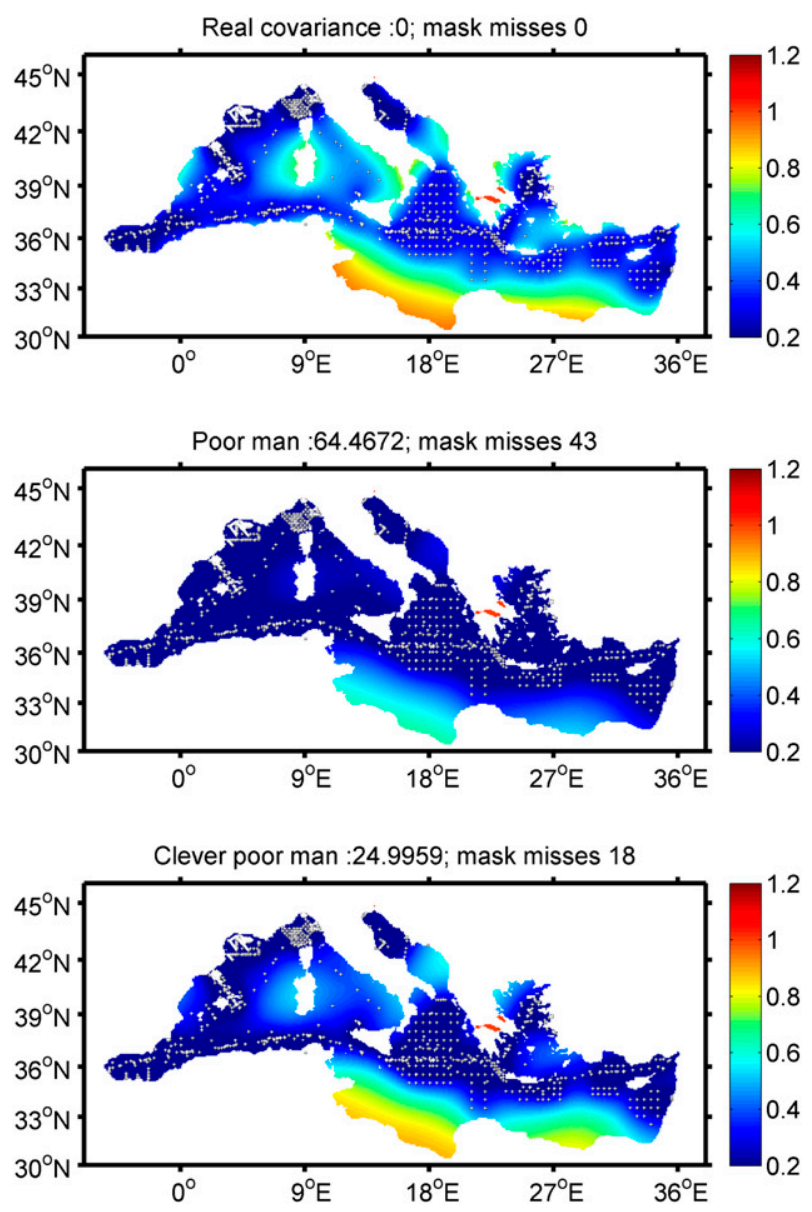

FIG. 7. Real error field, poor man's error, and clever poor man's error.

"almost exact" error field clearly better resolves features such as the higher error fields around Sardinia and in the eastern Thyrrenian Sea. Also, the error structure in the Alboran Sea is better recovered, despite the very low number of pseudodata (black dots) used.

For the error fields with boundary effects (Fig. 9), using the high pseudodata coverage along the coast makes it possible to capture the variable background variance, but because of the fine mesh along the coast, probably too many pseudodata have been added there. This results in excellent metrics, with only four incorrectly masked points and only $1 \%$ error in the error field. The relatively large number of pseudodata is then reflected also in the CPU time. But even with this coverage, the computational gain of a factor of 11 compared to the exact calculation is still significant. Comparing CPU times in this realistic case shows without doubt the usefulness of the new approaches (Table 1), which have been included in the DIVA tool (http://modb.oce.ulg.ac. be/mediawiki/index.php/DIVA). Indeed, climatology 

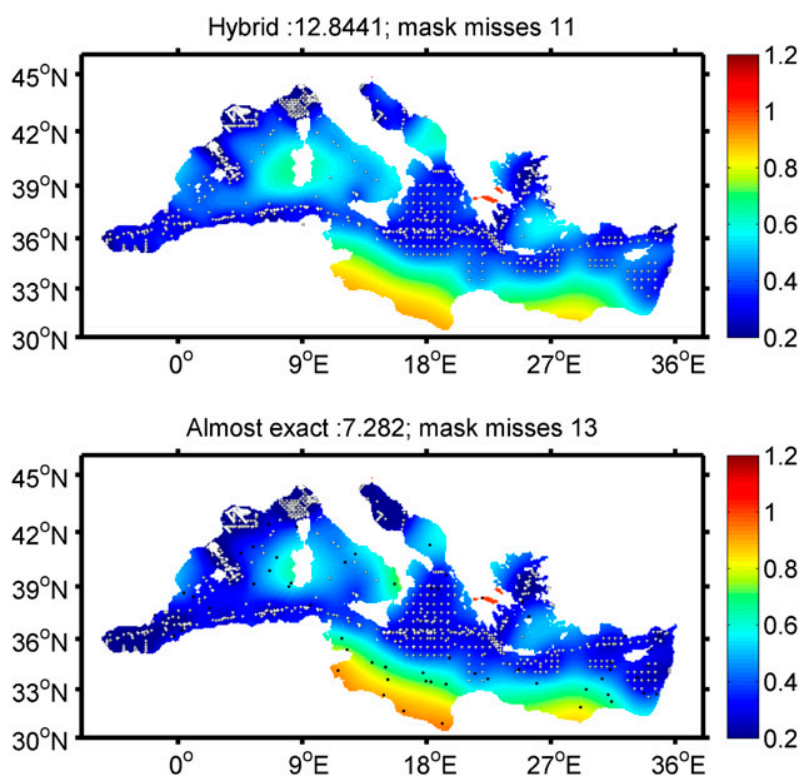

FIG. 8. Hybrid and almost exact approach.

productions generally require gridding at several levels, months, or seasons for several parameters, so that already in the $2 \mathrm{D}$ case the computational efficiency matters. When it comes to generalizations of our methods to 3DVAR or OI in several dimensions, the expected gain might be even more interesting as we will show now.

Here we presented some particular test cases and one may wonder how the computational efficiency behaves in other situations, in particular we want to know the computational gain we can expect for the almost exact error calculation compared to the exact one based on real covariances. In the latter case, for each grid point we need to perform an analysis. For an $n$-dimensional domain ${ }^{3}$ of size $D$ and grid spacing $\Delta$, the number of analysis for the exact error calculation is therefore $D / \Delta^{n}$. For the almost exact error calculation, we cover the domain with random points in which we need to make an analysis. This leads to $D / L^{n}$ required analyses, to which we have to add the analyses needed at the $N_{d}$ data locations. We evaluate this number as $\epsilon N_{d}$ with $\epsilon=1$ if we need to calculate $A_{i i}$ in all data points and $\epsilon<1$ if we use only a fraction $\epsilon$ of the observations to calculate the error exactly. We note that $\epsilon=0$ when a quality check approach using (17) already provided the values of $A_{i i}$. The gain $G$ we obtain is then written as

\footnotetext{
${ }^{3}$ We can assume that with a suitable change of the variable, the different dimensions have been made comparable.
}
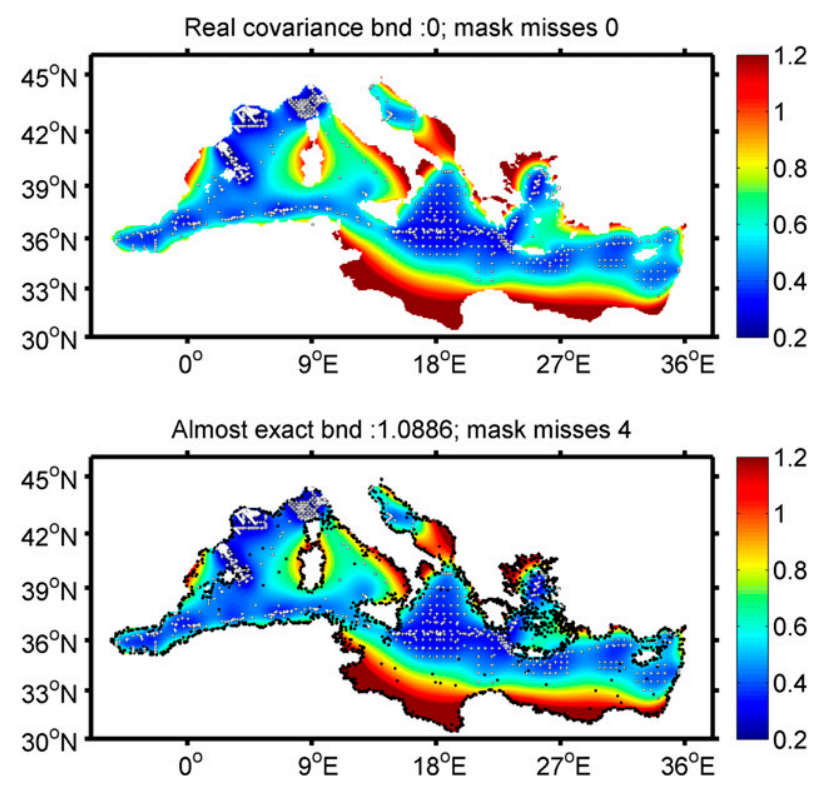

FIG. 9. Real error with boundary effects and almost exact approach.

$$
G=\frac{D / \Delta^{n}}{D / L^{n}+\epsilon N_{d}}=\frac{1}{1+\epsilon \frac{N_{d}}{\mathcal{N}}}\left(\frac{L}{\Delta}\right)^{n}
$$

where $\mathcal{N}=D / L^{n}$ is a measure of the degrees of freedom of the background field.

Normally, the numerical grids have a grid spacing that is much smaller than the physical length scales and the last term is therefore in favor of very high efficiency. If we work with a forecast model, its numerical grid is typically recommended to be 8 times smaller than the scales of interest. With only a few data points, we then reach gains of one to two orders of magnitude in $2 \mathrm{D}$ and almost three orders of magnitude in 3D. The gain decreases if the number of observations is high and allows for capturing the degrees of freedom of the system. If the number of observations is much larger than $\mathcal{N}$, then it is advised to use a fraction, $\epsilon \sim \mathcal{N} / \mathcal{N}_{d}$, to retain efficiency and still capture the error field.

\section{Generalizations}

We have presented our ideas in the framework of DIVA with a diagonal observational error covariance matrix and will now analyze how the methods can be applied in other frameworks.

One problem that can be encountered is therefore a nondiagonal observational error covariance matrix $\mathbf{R}$. The clever poor man's error was designed by looking at an isolated data point, and it was shown reliable in regions with isolated data and at sufficient distance from 
data clusters. In all these locations it does not matter whether $\mathbf{R}$ is diagonal and therefore the clever poor man's error should perform similarly in these regions. The application of the clever poor man's error by any analysis tool (be it OI, 3DVAR, or DIVA) technically also does not depend on whether $\mathbf{R}$ is diagonal, since it just demands the application of the analysis tool to a special data vector.

For the almost exact error, we notice that at data locations (7) still holds for nondiagonal $\mathbf{R}$ and that the value of the error at data location $i$ reads

$$
\mathbf{e}_{\mathbf{i}}^{\mathrm{T}} \mathbf{P}_{d} \mathbf{e}_{\mathbf{i}}=\mathbf{e}_{\mathbf{i}}^{\mathrm{T}} \underbrace{\mathbf{B} \mathbf{H}^{\mathrm{T}}\left(\mathbf{H B H}^{\mathrm{T}}+\mathbf{R}\right)^{-1}}_{\mathbf{K}} \mathbf{R} \mathbf{e}_{\mathbf{i}} .
$$

This can be read again from right to left to design the recipe for calculating the errors at the data locations: Extract column $i$ of the observational error covariance matrix (in other words, fill a vector with observational error covariances with respect to point $i$ ), apply your analysis tool $(\mathbf{K})$, and extract the solution at your data point $i$ (observation operator $\mathbf{e}_{\mathbf{i}}^{\mathrm{T}} \mathbf{H}$ ). If the tool does not rely on $\mathbf{R}$ but on its inverse, then an inversion is needed first, but as observational error covariance matrices are generally block diagonal with narrow bands, this is a feasible operation. Alternatively, one can calculate the error reduction term at location $i$ using

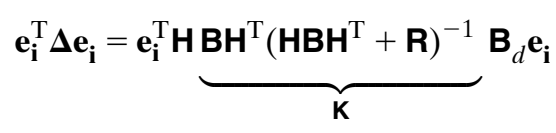

if it is easier to work with the background covariance matrix at the data points. We note that to apply (19), we actually do not even have to know exactly how the background error covariance is expressed; all we have to use is the analysis tool $\mathbf{K}$ applied to a series of data. One can therefore assess the exact error in a series of points and for the final gridding of the error, the tool can be used again, here even without the need to maintain the correlated observational error since the "field" to be gridded has no observational error anymore.

The presence of a nondiagonal $\mathbf{R}$ therefore still allows the application of the new methods by any analysis tool.

Another problem that can be encountered with tools other than DIVA is to find a way to adapt the correlation length during the clever poor man's error calculation or the final gridding step of the almost exact error approach. If the methods use a length scale in their formulation, then it should simply be adapted according to (11) for the specific correlation function of the method, so that the correlation function with the new scale mimics the squared correlation function. If the method uses an explicitly formulated correlation function that can be changed by the user, then it is suggested to replace the correlation function by its square. This is even simpler and further should improve the quality of the error field. This interpretation also paves the way for situations in which the background error covariance matrix is specified by numerical correlations. During the clever poor man's error calculation or the final gridding step of the almost exact error approach, one simply needs to use the squares of the correlations. In other cases, the background covariance can be formulated by recursive filters (e.g., Hayden and Purser 1995). Since these filters contain parameters determining the filter width, one can adapt the filter parameters to change the correlation length scales. Some other models work in spectral space and the analysis is also performed in spectral case. In these situations, the spectral representations of covariance functions have a specific signature of the correlation scales. Tampering with the spectrum can therefore be used to change the scales of the underlying covariance function-for example, a Gaussian correlation function in $n$ dimensions that only depends on distance $r$,

$$
c(r)=\exp \left(-r^{2} / L^{2}\right)
$$

has a spectral density $a(k)$ in wavenumber space $k$ given by

$$
a(k)=\left(\pi L^{2}\right)^{n / 2} \exp \left(-\pi^{2} L^{2} k^{2}\right) .
$$

To divide the length scale $L$ by a factor $\sqrt{2}$ in two dimensions, it is therefore sufficient to change amplitudes $a(k)$ according to

$$
a(k) \leftarrow \frac{a(0)}{2} \sqrt{\frac{a(k)}{a(0)}} .
$$

This shows that in order to reduce the correlation length scale, amplitudes of the higher modes get more importance. For spectral models on spheres, the coefficients of the spherical harmonics also define an underlying correlation function and can be modified to change the correlation length scale.

Still other background covariance specifications rely on projections on empirical orthogonal functions (EOFs). Such EOF decompositions are to some extent similar to a spectral decompositions, but the base functions are calculated from the data instead of being defined by analytical functions given a priori. The equivalent of the spectral density such as (22) is captured in the singular values of the singular value decomposition (SVD) 
leading to the EOFs. These coefficients or singular values can therefore be tampered with when a change in correlation length scale is to be obtained.

There are thus several possibilities to change the correlation function of the analysis tool so that it can be optimized to mimic its own square. In complicated implementations, the approach should, of course, be tested, and possibly calibrated, by looking at a covariance function generated by an analysis with a single data point and comparing it to the one obtained when using the tampered version. One should retrieve a correlation function for the tampered version, which is close to the square of the original one.

We see that there are many ways to adapt the length scale or correlations for the clever poor man' error calculation and the final gridding step of the almost exact error approach. Should this adaptation be difficult or not efficient, the almost exact error approach can still be applied by covering the domain with more pseudodata and making the final gridding step using the original covariances or a simpler gridding tool. Indeed, the error is already calculated exactly with a fine resolution so that any gridding method, even with a poorly specified correlation structure, when applied to these exact values of the error, should work fine. This is, however, then at the expense of more analyses to get the exact error in more locations.

To illustrate these ideas on an example, we can look at a typical 3DVAR approach used in operational mode, using the so-called National Meteorological Center (NMC) method (e.g., Parrish and Derber 1992; Fisher 2003), presented here, assuming we are still working with anomalies with respect to the background field.

It starts with a definition of a change of variables, with

$$
\mathbf{x}=\mathbf{U v}, \quad \mathbf{B}=\mathbf{U U}^{\mathrm{T}},
$$

so that (3) becomes

$$
2 J(\mathbf{v})=\mathbf{v}^{\mathrm{T}} \mathbf{v}+(\mathbf{H U v}-\mathbf{d})^{\mathrm{T}} \mathbf{R}^{-1}(\mathbf{H U v}-\mathbf{d}) .
$$

This can be interpreted as a change of variables in which the new state vector $\mathbf{v}$ has uncorrelated background errors. The minimization of (25) is then nicely conditioned if the observational error is large, since the quadratic function is then dominated by the background contribution, which is now isotropic and leading to good convergence. The fact that the solution is done via a new variable $\mathbf{v}$ is not essential for our purpose because after the minimization, the solution $\mathbf{x}=\mathbf{U} \mathbf{v}$ is still the minimum of (25) and hence defines the analysis tool $\mathbf{K}$ exactly as before. Also, the presence of a nondiagonal $\mathbf{R}$ causes no problem as shown earlier.
Therefore, the only problem we have to deal with is the problem of the correlation length scales or correlation functions, which need to be adapted. To do so, we now have to look at how $\mathbf{U}$ is designed. Written as

$$
\mathbf{v}=\mathbf{U}^{-1} \mathbf{x},
$$

we see that $\mathbf{U}^{-1}$ is supposed to transform the original state vector into one in which the background errors are uncorrelated. This supposes as a first step that physical balances are used to eliminate some variables as a function of others to avoid keeping the associated correlation. Formally, $\mathbf{U}_{p}^{-1} \mathbf{x}$ provides this state vector in which balances have been taken into account. At this step we do not need an adaptation for our methods. From there, the variables are scaled by the local standard deviation of the background field. For spectral methods this requires a transformation to real space, division by the local standard deviation, and a transformation back to spectral space, formally by applying $\Sigma^{-1}$. For a grid method, the operation simply divides by the local standard deviation. Here again, our methods do not introduce any change. Then the horizontal and vertical correlations need to be taken into account by successively trying to take out correlations in the horizontal (formally operation $\mathbf{U}_{h}^{-1}$ ) and the vertical directions (operation $\mathbf{U}_{v}^{-1}$ ). This will involve the correlations with which we will have to tamper. The operations now read as

$$
\mathbf{v}=\mathbf{U}_{v}^{-1} \mathbf{U}_{h}^{-1} \mathbf{\Sigma}^{-1} \mathbf{U}_{p}^{-1} \mathbf{x}
$$

which defines $\mathbf{U}=\mathbf{U}_{p} \boldsymbol{\Sigma} \mathbf{U}_{h} \mathbf{U}_{v}$ and finally $\mathbf{B}=$ $\mathbf{U}_{p} \boldsymbol{\Sigma} \mathbf{U}_{h} \mathbf{U}_{v} \mathbf{U}_{v}^{\mathrm{T}} \mathbf{U}_{h}^{\mathrm{T}} \boldsymbol{\Sigma}^{\mathrm{T}} \mathbf{U}_{p}^{\mathrm{T}}$. Obviously, in practice $\mathbf{B}$ is never formed but the succession of operations described above is applied to the state vector $\mathbf{x}$, then the minimization is performed on $\mathbf{v}$, and finally the optimal $\mathbf{x}$ is retrieved by the inverse operations in reverse order as shown:

$$
\mathbf{x}=\mathbf{U}_{p} \mathbf{\Sigma} \mathbf{U}_{h} \mathbf{U}_{v} \mathbf{v}
$$

There remains to be seen how to adapt $\mathbf{U}_{h}$ and $\mathbf{U}_{v}$ to accommodate changes in correlations. When the model works in spectral space, it is generally assumed that the modes are independent and matrix $\mathbf{U}_{h}$ is diagonal. The spectral coefficients found on the diagonal of $\mathbf{U}_{h}$ define in this case the underlying horizontal correlation function in physical space, and by changing their values, we can change the correlation function as shown in example (23). If the model works in grid space, then $\mathbf{U}_{h}$ can be specified by recursive filters or covariance functions, which can also be changed to meet our requirements. Finally, on the vertical, $\mathbf{U}_{v} \mathbf{U}_{v}^{\mathrm{T}}$ is in fact a vertical correlation 
matrix. It is generally considered a block diagonal matrix (one block for each variable and each spectral mode or spatial grid point) and is therefore composed of a series of small $N_{z} \times N_{z}$ correlation matrices, where $N_{z}$ is the number of vertical levels. These individual matrices must be adapted in our case to change the correlation functions. This can be done by taking, for example, the square of the correlations. If the matrices are already decomposed by a singular value decomposition to work with EOFs, as stated above, then one can tamper the singular values to change the correlations.

It is now clear that the adaptations to change the correlations are quite localized and therefore it should be possible to implement the poor man's error and the almost exact error calculations in operational 3DVAR implementations. We can finally note that in the NMC version, the parameters involved in $\mathbf{U}$ are fitted by assuming that statistics on differences in forecasts for the same moment but of different length ( 24 and $48 \mathrm{~h}$ ) are a good proxy for background errors. This calibration does not affect the possibility to readjust later the correlation during the poor man's error calculation or the final gridding of the almost exact error.

\section{Conclusions}

The preparation of error fields is generally much more expensive than the preparation of an analysis. We proposed two new ideas to provide some practical and economic ways to provide such error fields. The first method only needs a second analysis with modified correlation length scale and is particularly well suited for exploratory analysis or masking of gridded fields in regions insufficiently covered by data [such as done in the web version (Barth et al. 2010) or within Ocean Data View (ODV) (Schlitzer 2013)]. The second method, on the other hand, can be used for cases in which sufficient confidence in the covariance matrices justifies the use of the full error calculation. In this case, the new method we presented drastically reduces the computational burden without sacrificing the quality of the error field. The method is particularly useful when employed in parallel with outlier detection methods and cross validation, as the same computations can be reused.

We illustrated the approach using the specific analysis tool DIVA, but also paved the way for generalizations for a variety of situations when background covariances are formulated differently or when the observational error covariance matrix is nondiagonal. The ideas presented here can therefore be implemented in various versions of analysis tools.

In particular, we detailed how both methods can be adapted to 3DVAR approaches used in operational systems. They could then provide an alternative to the Lanczos vector-based estimates of the Hessian matrix. The new approach is particularly interesting if the background covariance is factorized or a very efficient preconditioning was applied so that the calculation of several minimizations to get error estimates in selected locations can be tackled.

Concerning future work in the context of DIVA, in the present paper we limited ourselves to the implementation of the case of uncorrelated observational error-that is, a diagonal R. Dealing with nondiagonal R is already more problematic with DIVA for the analysis itself. When data are provided with regular spatial patterns (such as along altimeter tracks or on satellite images), augmented data arrays can be used to account for correlated observational errors in methods that only deal with diagonal matrices for the observation error covariance (Brankart et al. 2009). This problem will be looked at in the future. Finally, there is also some room for improvement in DIVA in case one is interested in the unscaled error fields showing the boundary effects by reducing the number of pseudopoints near the boundaries if the computational load is too high and the meshes are very fine. The choice of the location of the additional pseudodata could also be further optimized when other constraints are used, such as the advection constraint already included in DIVA.

Acknowledgments. DIVA has received funding from the European Union Seventh Framework Programme (FP7/2007-2013) under Grant Agreement 283607, SeaDataNet 2, and from project EMODNET (MARE/2008/ 03-Lot 3 Chemistry-SI2.531432) from the DirectorateGeneral for Maritime Affairs and Fisheries. This research was also supported by the SANGOMA Project (European FP7-SPACE-2011 project, Grant 283580). The F.R.S.FNRS is acknowledged for providing supercomputing access. This is a MARE publication.

\section{REFERENCES}

Barth, A., A. Alvera-Azcárate, C. Troupin, M. Ouberdous, and J.-M. Beckers, 2010: A web interface for griding arbitrarily distributed in situ data based on Data-Interpolating Variational Analysis (DIVA). Adv. Geosci., 28, 29-37, doi:10.5194/ adgeo-28-29-2010.

— J.-M. Beckers, C. Troupin, A. Alvera-Azcárate, and L. Vandenbulcke, 2013: Divand-1.0: $n$-dimensional variational data analysis for ocean observations. Geosci. Model Dev. Discuss., 6, 4009-4051, doi:10.5194/gmdd-6-4009-2013.

Beckers, J.-M., A. Barth, and A. Alvera-Azcárate, 2006: DINEOF reconstruction of clouded images including error mapsApplication to the sea-surface temperature around Corsican Island. Ocean Sci., 2, 183-199, doi:10.5194/os-2-183-2006. 
Bekas, C., E. Kokiopoulou, and Y. Saad, 2007: An estimator for the diagonal of a matrix. Appl. Numer. Math., 57, 1214-1229.

Bouttier, F., and P. Courtier, 2002: Data assimilation concepts and methods March 1999. Meteorological Training Course Lecture Series, ECMWF, 59 pp. [Available online at http://www. ecmwf.int/newsevents/training/lecture_notes/pdf_files/ASSIM/ Ass_cons.pdf.]

Brankart, J.-M., and P. Brasseur, 1996: Optimal analysis of in situ data in the western Mediterranean using statistics and crossvalidation. J. Atmos. Oceanic Technol., 13, 477-491.

, C. Ubelmann, C.-E. Testut, E. Cosme, P. Brasseur, and J. Verron, 2009: Efficient parameterization of the observation error covariance matrix for square root or ensemble Kalman filters: Application to ocean altimetry. Mon. Wea. Rev., 137, 1908-1927.

Brasseur, P., 1994: Reconstruction de champs d'observations océanographiques par le modèle variationnel inverse: Méthodologie et applications. Ph.D. thesis, University of Liège, $262 \mathrm{pp}$.

_ J.-M. Beckers, J.-M. Brankart, and R. Schoenauen, 1996: Seasonal temperature and salinity fields in the Mediterranean Sea: Climatological analyses of a historical data set. Deep-Sea Res. I, 43, 159-192, doi:10.1016/0967-0637(96)00012-X.

Bretherton, F. P., R. E. Davis, and C. Fandry, 1976: A technique for objective analysis and design of oceanographic instruments applied to MODE-73. Deep-Sea Res., 23, 559-582, doi:10.1016/ 0011-7471(76)90001-2.

Courtier, P., J.-N. Thépaut, and A. Hollingsworth, 1994: A strategy for operational implementation of 4D-Var, using an incremental approach. Quart. J. Roy. Meteor. Soc., 120, 1367-1387, doi:10.1002/ qj. 49712051912.

Delhomme, J. P., 1978: Kriging in the hydrosciences. Adv. Water Resour., 1, 251-266, doi:10.1016/0309-1708(78)90039-8.

Emery, W. J., and R. E. Thomson, 2001: Data Analysis Methods in Physical Oceanography. 2nd ed. Elsevier, 654 pp.

Fischer, C., T. Montmerle, L. Berre, L. Auger, and S. E. Ştefănescu, 2005: An overview of the variational assimilation in the ALADIN/France numerical weather-prediction system. Quart. J. Roy. Meteor. Soc., 131, 3477-3492, doi:10.1256/qj.05.115.

Fisher, M., 2003: Background error covariance modelling. Seminar on Recent Development in Data Assimilation for Atmosphere and Ocean, Reading, United Kingdom, ECMWF, 45-63. [Available online at ftp://beryl.cerfacs.fr/pub/globc/exchanges/ daget/DOCS/sem2003_fisher.pdf.]

Gandin, L. S., 1965: Objective Analysis of Meteorological Fields. Israel Program for Scientific Translations, $242 \mathrm{pp}$.

Girard, D. A., 1998: Asymptotic comparison of (partial) crossvalidation, GCV and randomized GCV in nonparametric regression. Ann. Stat., 26, 315-334.

Hamill, T. M., and C. Snyder, 2000: A hybrid ensemble Kalman filter-3D variational analysis scheme. Mon. Wea. Rev., 128, 2905-2919.
Hayden, C. M., and R. J. Purser, 1995: Recursive filter objective analysis of meteorological fields: Applications to NESDIS operational processing. J. Appl. Meteor., 34, 3-15.

Kalnay, E., 2003: Atmospheric Modeling, Data Assimilation, and Predictability. Cambridge University Press, 341 pp.

Kaplan, A., Y. Kushnir, and M. A. Cane, 2000: Reduced space optimal interpolation of historical marine sea level pressure: 1854-1992. J. Climate, 13, 2987-3002.

Lorenc, A. C., 1986: Analysis methods for numerical weather prediction. Quart. J. Roy. Meteor. Soc., 112, 1177-1194, doi:10.1002/qj.49711247414.

McIntosh, P. C., 1990: Oceanographic data interpolation: Objective analysis and splines. J. Geophys. Res., 95 (C8), 13 529-13541.

Moore, A. M., H. G. Arango, G. Broquet, B. S. Powell, A. T. Weaver, and J. Zavala-Garay, 2011: The Regional Ocean Modeling System (ROMS) 4-dimensional variational data assimilation systems: Part I-System overview and formulation. Prog. Oceanogr., 91, 34-49, doi:10.1016/j.pocean.2011.05.004.

Parrish, D., and J. Derber, 1992: The National Meteorological Center's spectral statistical interpolation analysis system. Mon. Wea. Rev., 120, 1747-1763.

Rabier, F., and P. Courtier, 1992: Four-dimensional assimilation in the presence of baroclinic instability. Quart. J. Roy. Meteor. Soc., 118, 649-672, doi:10.1002/qj.49711850604.

Reynolds, R. W., and T. M. Smith, 1994: Improved global sea surface temperature analyses using optimum interpolation. J. Climate, 7, 929-948.

Rixen, M., J.-M. Beckers, J.-M. Brankart, and P. Brasseur, 2000: A numerically efficient data analysis method with error map generation. Ocean Modell., 2, 45-60, doi:10.1016/S1463-5003(00)00009-3.

Schlitzer, R., cited 2013: Ocean data view. [Available online at http://odv.awi.de.]

Seaman, R. S., and M. Hutchinson, 1985: Comparative real data tests of some objective analysis methods by withholding observations. Aust. Meteor. Mag., 33, 37-46.

Tang, J. M., and Y. Saad, 2012: A probing method for computing the diagonal of a matrix inverse. Numer. Linear Algebra Appl., 19, 485-501.

Troupin, C., F. Machín, M. Ouberdous, D. Sirjacobs, A. Barth, and J.-M. Beckers, 2010: High-resolution climatology of the north-east Atlantic using Data-Interpolating Variational Analysis (DIVA). J. Geophys. Res., 115, C08005, doi:10.1029/2009JC005512.

, and Coauthors, 2012: Generation of analysis and consistent error fields using the Data Interpolating Variational Analysis (DIVA). Ocean Modell., 52-53, 90-101, doi:10.1016/ j.ocemod.2012.05.002.

Wahba, G., and J. Wendelberger, 1980: Some new mathematical methods for variational objective analysis using splines and cross validation. Mon. Wea. Rev., 108, 1122-1143.

Xiang, D., and G. Wahba, 1996: A generalized approximate cross validation for smoothing splines with non-Gaussian data. Stat. Sin., 6, 675-692. 